\title{
Dietary polyunsaturated fatty acids and adaptation to chronic hypoxia alter acyl composition of serum and heart lipids
}

\author{
Patricie Balková ${ }^{1,2}$, Jana Ježková ${ }^{1}$, Markéta Hlaváčková ${ }^{1,3}$, Jan Neckáŕ2,4, Barbora Staňková ${ }^{5}$, \\ František Kolár $\check{r}^{2,4}$, František Novák ${ }^{2,3}$ and Olga Nováková ${ }^{1 *}$ \\ ${ }^{1}$ Department of Cell Biology, Faculty of Science, Charles University, Viničná 7128 00, Prague 2, Czech Republic \\ ${ }^{2}$ Centre for Cardiovascular Research, Prague, Czech Republic \\ ${ }^{3}$ Department of Biochemistry, Faculty of Science, Charles University, Prague, Czech Republic \\ ${ }^{4}$ Institute of Physiology, Academy of Sciences of the Czech Republic, Prague, Czech Republic \\ ${ }^{5}$ Fourth Department of Internal Medicine, First Faculty of Medicine, Charles University, Prague, Czech Republic
}

(Received 13 January 2009 - Revised 3 April 2009 - Accepted 16 April 2009 - First published online 1 June 2009)

\begin{abstract}
The effects of dietary supplementation with fat of different fatty acid profile and chronic intermittent hypoxia (CIH) on the fatty acid composition of serum and heart lipids were analysed. Adult male Wistar rats were fed a standard non-fat diet enriched with $10 \%$ of lard, fish oil ( $n$-3 PUFA) or maize oil ( $n-6$ PUFA) for 10 weeks. After 4 weeks on the diets, each group was divided in two subgroups, either exposed to CIH in a barochamber (7000 m, twenty-five exposures) or kept at normoxia. In normoxic rats, the fish oil diet increased the level of conjugated dienes. The $n$-6: $n$ - 3 PUFA ratio in serum TAG, phospholipids (PL), cholesteryl esters (CE) and heart TAG, PL and diacylglycerols (DAG) followed the ratio in the fed diet (in the sequence maize oil $>$ lard $>$ fish oil). In heart TAG, PL and DAG, 20:4n-6 and 18:2n-6 were replaced by $22: 6 n-3$ in the fish oil group. The main fatty acid in CE was $20: 4 n-6$ in the lard and maize oil groups whereas in the fish oil group, half of $20: 4 n-6$ was replaced by $20: 5 n-3$. CIH further increased $20: 5 n-3$ in CE in the fish oil group. CIH decreased the $n-6: n-3$ PUFA ratio in serum CE, heart TAG, PL and DAG in all dietary groups and stimulated the activity of catalase in the maize and fish oil groups. In conclusion, PUFA diets and CIH, both interventions considered to be cardioprotective, distinctly modified the fatty acid profile in serum and heart lipids with specific effects on conjugated diene production and catalase activity.
\end{abstract}

Dietary n-3 and n-6 PUFA: Chronic hypoxia: Serum and heart lipids: Antioxidative enzymes: Conjugated dienes: Cardioprotection

Lipids are important dietary components and changes in their quality may cause significant alterations in the fatty acid composition and properties of cardiac membranes. Changes in the composition of membrane lipids can account for nutritional benefits exerted by certain fatty acids through the so-called 'membrane lipid-therapy'(1). Many experimental and clinical studies have shown that dietary lipids can influence the incidence and severity of CHD. While MUFA and PUFA (n-3 and $n-6$ classes) usually display beneficial effects, SFA may have an adverse impact on the heart ${ }^{(2-4)}$. Both classes of PUFA collectively protect the heart against ischaemia-reperfusion injury but $n$-3 PUFA are considered more potent in their cardioprotective activity ${ }^{(5)}$. Dietary PUFA play a key role in cardiac protection by modulating serum and tissue lipid composition and in this regard affecting metabolic and signal-transduction pathways ${ }^{(6)}$. Chronic intermittent hypoxia $(\mathrm{CIH})$ is another protective phenomenon that increases cardiac tolerance to all major damaging consequences of ischaemiareperfusion $^{(7)}$. Our recent results suggest that the signalling pathway involving protein kinase $\mathrm{C}$ (PKC) and reactive oxygen species plays a role in the mechanism of $\mathrm{CIH}$-induced cardioprotection $^{(8)}$. Moreover, we have shown that $\mathrm{CIH}$ induces the remodelling of cardiac membranes leading to the increase in the $n-3$ PUFA proportion in phospholipids (PL) ${ }^{(9)}$.

The present study was prompted by results of our recent experiments $^{(10)}$ combining two systemic interventions that can improve cardiac ischaemic tolerance: dietary supplementation with $n-3$ or $n-6$ PUFA and adaptation to CIH. The $n-6$ and $n$-3 PUFA-enriched diets as well as $\mathrm{CIH}$ protected against arrhythmias but had distinct effects on myocardial infarct size induced by coronary artery occlusion. The $n-6: n-3$ PUFA ratio in the heart PL was altered in accordance with the fatty acid composition of the lipid diet while $\mathrm{CIH}$ decreased this ratio to the same extent, independent of dietary fatty acid profile. The main purpose of the present study was to analyse in detail the effects of lipid diets and $\mathrm{CIH}$ on acyl composition of the main lipid classes in rat serum and heart. For the first time we demonstrated that, under these conditions, the individual classes of transport and membrane lipids markedly differ in their ability to maintain fatty acid composition. In line

Abbreviations: CE, cholesteryl ester; CIH, chronic intermittent hypoxia; DAG, diacylglycerol; GPX, glutathione peroxidase; PKC, protein kinase C; PL, phospholipid; SOD, superoxide dismutase.

* Corresponding author: Dr Olga Nováková, fax +420 221951758, email olnov@ natur.cuni.cz 
with the increased susceptibility of $n-3$ PUFA to oxidative stress, myocardial markers of lipid peroxidation increased in fish oil-supplemented rats.

\section{Materials and methods}

\section{Animal model}

Adult male Wistar rats (weight 250-280 g) were fed a non-fat standard ST1 diet (Velaz, Czech Republic) enriched with either $10 \%$ by weight of lard, fish oil ( $n$-3 PUFA; Löhmann \& Voss, Germany) or maize oil (n-6 PUFA; Olmühle $\mathrm{GmbH}$, Austria) for 10 weeks. The composition of the nonfat standard ST1 diet was as follows (per $\mathrm{kg}$ ): $240 \mathrm{~g}$ crude protein; $37.2 \mathrm{~g}$ non-nutrient fibres; $65.1 \mathrm{~g}$ ash; $240 \mathrm{~g} \mathrm{~N}$ substances; antioxidants (butylhydroxytoluene, ethoxyquin, butylhydroxyanisol); $4500 \mu \mathrm{g}$ (15000 IU) vitamin A; $25 \mu \mathrm{g}$ (1000 IU) vitamin D; $107.9 \mathrm{mg}$ vitamin E; $0.34 \mathrm{mg} \mathrm{Se}$; $22.6 \mathrm{mg} \mathrm{Cu} ; 13.2 \mathrm{~g} \mathrm{Ca} ; 8.7 \mathrm{~g} \mathrm{P} ; 1.8 \mathrm{~g} \mathrm{Na}$; Myco ad (adsorbent of mycotoxins). The fatty acid composition of the diets enriched with lard, fish oil or maize oil is shown in Table 1; the term 'enriched' is omitted hereafter for the sake of simplicity. After 4 weeks on the diets, each group was divided into two subgroups that were either exposed or not to chronic intermittent (hypobaric) hypoxia corresponding to $7000 \mathrm{~m}$ for $8 \mathrm{~h} / \mathrm{d}, 5 \mathrm{~d} /$ week. In the exposed subgroups, barometric pressure $\left(P_{\mathrm{B}}\right)$ was lowered stepwise so that the level equivalent to an altitude of $7000 \mathrm{~m}\left(P_{\mathrm{B}}=41 \mathrm{kPa} ; P_{2}=8.6 \mathrm{kPa}\right)$ was reached after thirteen exposures; the total number of exposures was twenty-five ${ }^{(8)}$. The control subgroups of animals were kept for the same period of time at $P_{\mathrm{B}}$ and $\mathrm{PO}_{2}$ equivalent to an altitude of $200 \mathrm{~m}\left(P_{\mathrm{B}}=99 \mathrm{kPa} ; P_{2}=20.7 \mathrm{kPa}\right)$. All animals had free access to water. The day after the last hypoxic exposure, rats were anaesthetised by sodium pentobarbitone $(60 \mathrm{mg} / \mathrm{kg}$ body weight, intraperitoneally; Sanofi, France).

Table 1. Fatty acid composition ( $\mathrm{mol} \%$ ) of the diet lipids

(Means of two separate analyses)

\begin{tabular}{|c|c|c|c|}
\hline & Lard & Fish oil & Maize oil \\
\hline $14: 0$ & 1.40 & $5 \cdot 66$ & 0.58 \\
\hline $16: 0$ & $24 \cdot 15$ & $17 \cdot 86$ & $12 \cdot 28$ \\
\hline $16: 1 n-7$ & $2 \cdot 48$ & $7 \cdot 71$ & 0.93 \\
\hline $18: 0$ & $10 \cdot 87$ & $2 \cdot 13$ & 1.81 \\
\hline $18: 1 n-9$ & $35 \cdot 50$ & $16 \cdot 48$ & $24 \cdot 70$ \\
\hline $18: 1 n-7$ & 2.49 & 3.44 & 0.95 \\
\hline $18: 2 n-6$ & $19 \cdot 03$ & $15 \cdot 38$ & $53 \cdot 16$ \\
\hline $18: 3 n-3$ & 1.62 & $2 \cdot 61$ & $2 \cdot 31$ \\
\hline $20: 1 n-9$ & 0.96 & $7 \cdot 31$ & 1.03 \\
\hline $20: 4 n-6$ & $0 \cdot 10$ & 0.49 & 0.05 \\
\hline $20: 5 n-3$ & 0.18 & 9.94 & ND \\
\hline $22: 5 n-3$ & 0.07 & 1.78 & ND \\
\hline $22: 6 n-3$ & 0.21 & 8.04 & ND \\
\hline $20: 4 n-6: 22: 6 n-3$ ratio & 0.47 & 0.06 & ND \\
\hline$\Sigma \mathrm{SFA}^{*}$ & $36 \cdot 42$ & $25 \cdot 65$ & $14 \cdot 67$ \\
\hline$\Sigma$ MUFA* $^{*}$ & 41.43 & 34.94 & $27 \cdot 61$ \\
\hline$\sum n-6$ PUFA* & $19 \cdot 30$ & $15 \cdot 38$ & $53 \cdot 16$ \\
\hline$\sum n-3$ PUFA* & 2.08 & $22 \cdot 70$ & $2 \cdot 31$ \\
\hline Unsaturation index & $86 \cdot 86$ & $180 \cdot 37$ & $140 \cdot 86$ \\
\hline
\end{tabular}

ND, not detected.

${ }^{*} \Sigma$ SFA, $\Sigma$ MUFA, $\Sigma n-6$ PUFA and $\Sigma n-3$ PUFA comprise of all fatty acids analysed.

†The unsaturation index is calculated as mol \% of individual unsaturated fatty acids multiplied by the number of double bonds.
After collecting the blood samples, the hearts were rapidly excised, washed in cold $\left(0^{\circ} \mathrm{C}\right)$ saline and dissected into the right and left ventricular free walls and the septum. Heart regions were weighed and the left ventricles were frozen and stored in liquid $\mathrm{N}_{2}$. All of the chemicals were purchased from Sigma (St Louis, MO, USA), unless otherwise indicated. The study was conducted in accordance with the Guide for the Care and Use of Laboratory Animals published by the US National Institutes of Health (NIH publication no. 85-23, revised 1996). The experimental protocol was approved by the Animal Care and Use Committee of the Institute of Physiology, Academy of Sciences of the Czech Republic.

\section{Fatty acid composition}

Serum ( $1 \mathrm{ml}$ ) was dissolved in $21 \mathrm{ml}$ of a chloroform-methanol mixture $(2: 1, \mathrm{v} / \mathrm{v})$ and shaken in a pear-shaped flask. The serum protein precipitate was removed by filtration. Then $10 \mathrm{ml}$ of a chloroform-methanol-water mixture (3:48:47, by vol.) was added and after a vigorous shaking the lower lipid layer was separated and dried at $40^{\circ} \mathrm{C}$ under a stream of $\mathrm{N}_{2}$. Individual lipids, i.e. PL, TAG and cholesteryl esters (CE), were separated by onedimensional TLC $(0.5 \mathrm{~mm}$ Silica Gel H; Merck, Darmstadt, Germany) using the solvent mixture hexane-diethyl etheracetic acid (85:15:1, by vol.), detected by 2,7 dichlorofluorescein $\left(0.005 \%\right.$ in methanol), scraped out and stored in an $\mathrm{N}_{2}$ atmosphere at $-20^{\circ} \mathrm{C}$. On the next day, fatty acid methyl esters were prepared and separated by $\mathrm{GC}^{(11)}$.

Heart lipids and their fatty acid composition were analysed as described previously ${ }^{(9)}$. Briefly, frozen left ventricular myocardium was pulverised and homogenised. Lipids were extracted according to Folch et al. ${ }^{(12)}$ and dried at $40^{\circ} \mathrm{C}$ under a stream of $\mathrm{N}_{2}$. The one-dimensional TLC of PL, TAG and diacylglycerols (DAG) as well as further procedures were the same as with the serum samples.

\section{Antioxidative enzyme activities}

Frozen left ventricular myocardium was pulverised to a fine powder at the temperature of liquid $\mathrm{N}_{2}$, followed by PotterElvehjem homogenisation in 8 volumes of ice-cold buffer composed of (mmol/l): 12.5 2-amino-2-hydroxymethylpropane-1,3-diol (Tris)-HCl (pH 7.4); 250 sucrose; 2.5 ethylene glycol tetra-acetic acid; 1 EDTA; $100 \mathrm{NaF} ; 5$ dithiothreitol; 0.3 phenylmethylsulfonyl fluoride; $0 \cdot 2$ leupeptin; 0.02 aprotinin.

Catalase activity was measured by the method of Aebi ${ }^{(13)}$. The rate of $\mathrm{H}_{2} \mathrm{O}_{2}$ decomposition was monitored spectrophotometrically.

Glutathione peroxidase (GPX) activity was determined by the indirect procedure described by Paglia \& Valentine ${ }^{(14)}$. Oxidised glutathione was produced by GPX reaction and immediately reduced by NADPH in the presence of glutathione reductase. The rate of NADPH consumption was recorded as a measure of oxidised glutathione formation.

Total superoxide dismutase (SOD) activity was determined by the modified nitroblue tetrazolium method in homogenates $^{(15)}$. The xanthine-xanthine oxidase reaction was utilised to generate a superoxide flux. Nitroblue tetrazolium reduction by superoxide anions to blue formazan was measured spectrophotometrically. 


\section{Conjugated dienes}

The extraction of lipids was carried out by the method of Folch et al. as described above ${ }^{(12)}$. The dry lipid residue was redissolved in cyclohexane and the tissue content of conjugated dienes was measured spectrophotometrically ${ }^{(16)}$.

\section{Statistical analysis}

The results are expressed as mean values with their standard errors. Two-way ANOVA and subsequent StudentNewman-Keuls tests were used for comparison of differences in normally distributed variables between groups. Differences were considered as statistically significant when $P<0 \cdot 05$.

\section{Results}

Fatty acid composition of serum and heart lipids

Table 2 presents fatty acid composition of serum lipids. The fatty acid profiles and values of the unsaturation index in serum TAG of normoxic dietary groups closely followed those of the corresponding fed diets (Table 1). The proportion of SFA and MUFA decreased in favour of total PUFA in the

Table 2. Effect of diets and chronic hypoxia on the fatty acid composition in serum lipids

(Mean values with their standard errors for five animals per group)

\begin{tabular}{|c|c|c|c|c|c|c|c|c|c|c|c|c|}
\hline & \multicolumn{6}{|c|}{ Normoxia } & \multicolumn{6}{|c|}{ Hypoxia } \\
\hline & \multicolumn{2}{|c|}{ Lard } & \multicolumn{2}{|c|}{ Fish oil } & \multicolumn{2}{|c|}{ Maize oil } & \multicolumn{2}{|c|}{ Lard } & \multicolumn{2}{|c|}{ Fish oil } & \multicolumn{2}{|c|}{ Maize oil } \\
\hline & Mean & SEM & Mean & SEM & Mean & SEM & Mean & SEM & Mean & SEM & Mean & SEM \\
\hline \multicolumn{13}{|l|}{ TAG } \\
\hline $16: 0$ & 29.02 & 0.39 & $23 \cdot 67 \S$ & 0.82 & $17 \cdot 27 \S$ & 0.59 & 30.57 & 0.48 & $23.83 \S$ & 0.38 & $21 \cdot 23 \S^{*}$ & 0.73 \\
\hline $18: 0$ & 5.43 & 0.37 & $2 \cdot 20 \dagger$ & 0.09 & $1.67 \dagger$ & 0.12 & $5 \cdot 61$ & 0.36 & $2.42 \dagger$ & 0.24 & $1.72 \dagger$ & 0.06 \\
\hline $18: 1 n-9$ & $37 \cdot 34$ & 0.47 & 23.79† & 0.81 & $24.45 \dagger$ & 0.65 & $39 \cdot 20$ & 0.63 & $24 \cdot 24 \S$ & 0.77 & $27 \cdot 69 \S^{*}$ & 0.49 \\
\hline $18: 2 n-6$ & $16 \cdot 03$ & 0.27 & $15 \cdot 21$ & 0.73 & $44 \cdot 10 \S$ & $1 \cdot 30$ & $12 \cdot 72^{*}$ & 0.98 & $14 \cdot 88$ & 0.69 & $38.73 \S^{\star}$ & 1.06 \\
\hline $20: 4 n-6$ & $1 \cdot 27$ & 0.08 & $0.62 \S$ & 0.02 & $3.98 \S$ & 0.33 & 0.88 & 0.17 & 0.68 & 0.06 & $2 \cdot 34 \S^{\star}$ & 0.26 \\
\hline $20: 5 n-3$ & 0.24 & 0.01 & $6 \cdot 01 \S$ & 0.61 & 0.25 & 0.05 & 0.13 & 0.03 & $6 \cdot 15 \S$ & 0.68 & 0.14 & 0.03 \\
\hline $22: 6 n-3$ & 0.83 & 0.05 & $10 \cdot 04 \S$ & 0.54 & 0.73 & 0.13 & 0.50 & 0.09 & $9 \cdot 22 \S$ & 0.78 & 0.31 & 0.04 \\
\hline $20: 4 n-6: 22: 6 n-3$ ratio & 1.54 & 0.11 & 0.06 & 0.004 & $6 \cdot 12 \S$ & 0.92 & 1.91 & 0.25 & $0.07 \S$ & 0.01 & $7 \cdot 62 \S$ & 0.74 \\
\hline$\Sigma$ SFA\| & $35 \cdot 61$ & 0.53 & $27 \cdot 77 \S$ & 0.79 & $19 \cdot 63 \S$ & 0.61 & $37 \cdot 24$ & 0.40 & $28 \cdot 08 \S$ & 0.44 & $23.59 \S^{*}$ & 0.74 \\
\hline$\Sigma$ MUFA\| & 43.49 & 0.49 & $35 \cdot 24 \S$ & $1 \cdot 17$ & $27 \cdot 55 \S$ & 0.80 & $46 \cdot 47$ & $1 \cdot 29$ & $36 \cdot 31 \S$ & 1.02 & $32 \cdot 03 \S^{*}$ & 0.40 \\
\hline$\sum n-6$ PUFA\| & $18 \cdot 52$ & 0.21 & $16 \cdot 49$ & 0.76 & $50 \cdot 57 \S$ & 1.08 & $14 \cdot 83^{\star}$ & $1 \cdot 32$ & $16 \cdot 23$ & 0.75 & $42.97 \S^{*}$ & 0.73 \\
\hline$\sum n-3$ PUFA\| & $2 \cdot 36$ & 0.10 & $20 \cdot 48 \S$ & $1 \cdot 15$ & $2 \cdot 24$ & 0.24 & 1.44 & 0.22 & $19 \cdot 36 \S$ & 1.59 & 1.40 & 0.10 \\
\hline Unsaturation index & $95 \cdot 75$ & $1 \cdot 15$ & $180 \cdot 57 \S$ & $4 \cdot 13$ & $150 \cdot 38 \S$ & $2 \cdot 37$ & 86.06 & $3 \cdot 60$ & $175 \cdot 02 \S$ & $6 \cdot 39$ & $131 \cdot 17 \S^{*}$ & $1 \cdot 11$ \\
\hline \multicolumn{13}{|l|}{ Phospholipids } \\
\hline $16: 0$ & $20 \cdot 30$ & 0.25 & $24.48 \dagger$ & 0.82 & $23 \cdot 17 \dagger$ & 0.79 & $22 \cdot 49^{\star}$ & 0.45 & $28 \cdot 14 \S^{*}$ & 0.59 & $22 \cdot 38$ & 0.34 \\
\hline $18: 0$ & $23 \cdot 86$ & 0.78 & $20.01 \dagger$ & 0.76 & $19.55 \dagger$ & $1 \cdot 26$ & 24.93 & $2 \cdot 67$ & $18 \cdot 34 \dagger$ & $1 \cdot 24$ & $19 \cdot 05 \dagger$ & 0.70 \\
\hline $18: 1 n-9$ & $6 \cdot 27$ & 0.25 & 5.64 & 0.50 & 5.06 & 0.56 & $8 \cdot 14^{\star}$ & 0.48 & $6.58 \S$ & 0.23 & $5 \cdot 32 \S$ & 0.26 \\
\hline $18: 2 n-6$ & 23.01 & 0.73 & $19 \cdot 35$ & 1.79 & 22.97 & $1 \cdot 33$ & $22 \cdot 82$ & 0.33 & $18.56 \S$ & $1 \cdot 13$ & $28 \cdot 40 \S^{*}$ & 0.52 \\
\hline $20: 4 n-6$ & $19 \cdot 47$ & $1 \cdot 15$ & $11.89 \S$ & $2 \cdot 85$ & $20 \cdot 24$ & 1.46 & $14 \cdot 49^{*}$ & 0.26 & $6 \cdot 89 \S$ & 0.36 & $18 \cdot 83 \S$ & 0.58 \\
\hline $20: 5 n-3$ & 0.23 & 0.03 & $5.91 \S$ & 1.50 & 0.64 & 0.50 & 0.24 & 0.05 & $8.02 \S$ & 0.15 & 0.08 & 0.02 \\
\hline $22: 6 n-3$ & $2 \cdot 30$ & 0.16 & $4 \cdot 42 \S$ & 0.77 & $2 \cdot 51$ & 0.45 & $2 \cdot 28$ & 0.09 & $5.46 \S$ & 0.37 & 1.94 & 0.09 \\
\hline $20: 4 n-6: 22: 6 n-3$ ratio & $8 \cdot 61$ & 0.54 & 2.69 & $2 \cdot 10$ & 8.06 & 1.94 & $6 \cdot 52^{*}$ & 0.21 & $1 \cdot 26 \S$ & $0 \cdot 12$ & $9 \cdot 71 \S$ & 0.76 \\
\hline$\Sigma$ SFA\| & $44 \cdot 49$ & 0.70 & $42 \cdot 34$ & 0.81 & $43 \cdot 24$ & $1 \cdot 77$ & 44.90 & 0.37 & 46.05 & 1.42 & $42 \cdot 72$ & $1 \cdot 42$ \\
\hline$\Sigma$ MUFA\| & $8 \cdot 39$ & 0.22 & $9 \cdot 90$ & $1 \cdot 12$ & $8 \cdot 40$ & 1.06 & $10 \cdot 56^{\star}$ & 0.55 & $11 \cdot 72$ & 0.31 & $7 \cdot 51 \S$ & 0.31 \\
\hline$\sum n-6$ PUFA $\|$ & $44 \cdot 05$ & 0.74 & 35.42 & 4.06 & $45 \cdot 84$ & $2 \cdot 40$ & $42 \cdot 07$ & 0.45 & $27 \cdot 56 \S$ & 0.97 & $47 \cdot 62$ & 1.46 \\
\hline$\sum n-3$ PUFA\| & 3.07 & 0.16 & $11.97 \S$ & $2 \cdot 31$ & $3 \cdot 26$ & 0.99 & 2.98 & 0.11 & $14.66 \S$ & 0.40 & $2 \cdot 37$ & 0.08 \\
\hline Unsaturation index & $154 \cdot 46$ & 4.06 & $171 \cdot 28 \dagger$ & $1 \cdot 30$ & $161 \cdot 46$ & $4 \cdot 80$ & $142 \cdot 28^{*}$ & 1.50 & $160 \cdot 24^{*}$ & $4 \cdot 30$ & $152 \cdot 12$ & $6 \cdot 44$ \\
\hline \multicolumn{13}{|l|}{ Cholesteryl esters } \\
\hline $16: 0$ & 8.57 & 0.67 & $10 \cdot 71$ & 0.72 & $7 \cdot 53 \ddagger$ & 0.59 & 9.83 & 0.33 & 11.05 & 0.39 & $9 \cdot 33$ & 1.40 \\
\hline $18: 0$ & 1.03 & 0.18 & 1.36 & 0.34 & 0.82 & 0.27 & $1 \cdot 17$ & 0.11 & $1 \cdot 23$ & $0 \cdot 14$ & 1.42 & 0.59 \\
\hline $18: 1 n-9$ & $7 \cdot 57$ & 0.23 & $8 \cdot 11$ & 0.25 & $8 \cdot 14$ & $1 \cdot 16$ & $8 \cdot 92$ & 0.40 & $7 \cdot 90$ & $0 \cdot 11$ & $12 \cdot 71$ & $4 \cdot 68$ \\
\hline $18: 2 n-6$ & $26 \cdot 52$ & 0.80 & $22 \cdot 31 \S$ & $1 \cdot 70$ & $28 \cdot 34$ & 0.68 & $27 \cdot 65$ & $1 \cdot 20$ & $19 \cdot 03 \S$ & 0.47 & $29 \cdot 43$ & 1.60 \\
\hline $20: 4 n-6$ & $50 \cdot 15$ & 1.64 & $24 \cdot 37 \S$ & $5 \cdot 82$ & $49 \cdot 81$ & $2 \cdot 62$ & $44 \cdot 84$ & $2 \cdot 00$ & $16 \cdot 55 \S$ & 0.27 & $40 \cdot 88$ & $5 \cdot 36$ \\
\hline $20: 5 n-3$ & 0.54 & 0.13 & $23 \cdot 41 \S$ & $5 \cdot 72$ & 0.36 & 0.13 & 0.53 & 0.18 & $33.74 \S^{*}$ & 0.54 & 0.23 & 0.05 \\
\hline $22: 6 n-3$ & 0.72 & 0.02 & $1.62 \S$ & 0.24 & 0.50 & 0.04 & 0.86 & 0.06 & $1.62 \S$ & 0.11 & 0.61 & 0.09 \\
\hline $20: 4 n-6: 22: 6 n-3$ ratio & $70 \cdot 00$ & 0.88 & $20 \cdot 14 \S$ & $9 \cdot 78$ & $100 \cdot 97 \S$ & $7 \cdot 87$ & 53.09 & $3 \cdot 24$ & $10 \cdot 47 \S$ & 0.99 & $68 \cdot 97^{*}$ & $9 \cdot 39$ \\
\hline$\Sigma$ SFA\| & $10 \cdot 18$ & 0.86 & $13 \cdot 01$ & 0.98 & $9 \cdot 08 \ddagger$ & $1 \cdot 12$ & $11 \cdot 87$ & 0.32 & $13 \cdot 33$ & 0.37 & 11.52 & 1.85 \\
\hline$\Sigma$ MUFA $\|$ & $10 \cdot 84$ & 0.62 & 14.06 & 1.07 & $10 \cdot 77$ & $2 \cdot 02$ & $12 \cdot 70$ & 0.58 & 14.49 & 0.33 & $15 \cdot 83$ & $5 \cdot 32$ \\
\hline$\sum n-6$ PUFA\| & $77 \cdot 46$ & $1 \cdot 30$ & $47 \cdot 34 \S$ & $7 \cdot 53$ & $79 \cdot 30$ & $3 \cdot 23$ & 73.68 & 0.82 & $36 \cdot 44 \S$ & 0.35 & $71 \cdot 38$ & $6 \cdot 88$ \\
\hline$\sum n$-3 PUFA\| & 1.53 & 0.12 & $25 \cdot 50 \S$ & 5.96 & $1 \cdot 11$ & 0.17 & $1 \cdot 73$ & 0.13 & $35 \cdot 73 \S^{*}$ & 0.57 & $1 \cdot 26$ & 0.12 \\
\hline Unsaturation index & 274.68 & $5 \cdot 38$ & $286 \cdot 47$ & $6 \cdot 52$ & 274.99 & $9 \cdot 42$ & $259 \cdot 85$ & $4 \cdot 86$ & $301 \cdot 16 \S$ & 1.92 & $247 \cdot 73$ & $19 \cdot 49$ \\
\hline
\end{tabular}

${ }^{*}$ Mean value was significantly different from that of the corresponding normoxic group $(P<0.05)$.

† Mean value was significantly different from that of the corresponding lard dietary group $(P<0.05)$.

$\ddagger$ Mean value was significantly different from that of the corresponding fish oil dietary group $(P<0.05)$.

$\S$ Mean value was significantly different from those of the other dietary groups $(P<0.05)$

$\| \Sigma$ SFA, $\Sigma$ MUFA, $\Sigma n$-6 PUFA and $\Sigma n$-3 PUFA comprise of all fatty acids analysed.

I The unsaturation index is calculated as mol \% of individual unsaturated fatty acids multiplied by the number of double bonds. 
following sequence: lard $>$ fish oil $>$ maize oil dietary groups. The high abundance of $18: 1 n-9$ was the characteristic feature of the lard dietary group whereas the maize oil group was rich in $18: 2 n-6$. In contrast to the fed diets, $20: 4 n-6$ appeared in serum TAG in the lard and particularly in the maize oil dietary groups. The highest unsaturation index of the fish oil group was in agreement with the markedly higher $n-3$ PUFA proportion in this group compared with other dietary groups. CIH increased SFA and MUFA proportions due to the rise of $16: 0$ and $18: 1 n-9$ and decreased the $n-6$ PUFA proportion due to the fall of $18: 2 n-6$ and
$20: 4 n-6$ in the maize oil dietary group, resulting in lowered unsaturation index values. Regarding the lard group, $\mathrm{CIH}$ decreased the $18: 2 n-6$ proportion only. No effect of $\mathrm{CIH}$ on serum TAG was observed in the fish oil group.

The dominant fatty acids in serum PL of the lard and maize oil dietary groups were $16: 0,18: 0,18: 2 n-6$ and $20: 4 n-6$ in nearly equimolar proportions while for the fish oil group, a half of $20: 4 n-6$ was substituted by $20: 5 n-3$ and $22: 6 n-3$. $\mathrm{CIH}$ increased the abundance of $16: 0$ and $18: 1 n-9$ and decreased $20: 4 n-6$ in serum PL of the lard group with a qualitatively similar but less pronounced effect in the fish oil group;

Table 3. Effect of diets and chronic hypoxia on the fatty acid composition in heart lipids (Mean values with their standard errors for five animals per group)

\begin{tabular}{|c|c|c|c|c|c|c|c|c|c|c|c|c|}
\hline & \multicolumn{6}{|c|}{ Normoxia } & \multicolumn{6}{|c|}{ Hypoxia } \\
\hline & \multicolumn{2}{|c|}{ Lard } & \multicolumn{2}{|c|}{ Fish oil } & \multicolumn{2}{|c|}{ Maize oil } & \multicolumn{2}{|c|}{ Lard } & \multicolumn{2}{|c|}{ Fish oil } & \multicolumn{2}{|c|}{ Maize oil } \\
\hline & Mean & SEM & Mean & SEM & Mean & SEM & Mean & SEM & Mean & SEM & Mean & SEM \\
\hline \multicolumn{13}{|l|}{ TAG } \\
\hline $16: 0$ & 27.39 & 0.76 & $22 \cdot 74 \dagger$ & 0.52 & $19 \cdot 84 \dagger$ & 1.42 & $27 \cdot 61$ & 1.56 & $21 \cdot 16 \dagger$ & 1.58 & $17 \cdot 83 \dagger$ & 0.81 \\
\hline $18: 0$ & $10 \cdot 78$ & 0.42 & $4.94 \dagger$ & 0.22 & $5.99 \dagger$ & 0.73 & $10 \cdot 71$ & 0.69 & $4.38 \dagger$ & 0.22 & $5 \cdot 14 \dagger$ & 0.73 \\
\hline $18: 1 n-9$ & 34.9 & 0.80 & $25 \cdot 80 \dagger$ & 0.87 & $25.59 \dagger$ & 0.78 & 32.40 & 1.08 & $23 \cdot 18 \dagger^{\star}$ & 0.57 & $25.46 \dagger$ & 0.51 \\
\hline $18: 2 n-6$ & 13.58 & 0.26 & $16 \cdot 59 \S$ & 0.65 & $37.83 \S$ & 1.22 & $12 \cdot 92$ & 0.42 & $15 \cdot 39 \S$ & 0.38 & $39.93 \S$ & 1.01 \\
\hline $20: 4 n-6$ & 1.46 & 0.11 & 0.73 & 0.05 & $2 \cdot 28 \S$ & 0.30 & $1 \cdot 76$ & 0.16 & $0.78 \S$ & 0.05 & 2.08 & 0.36 \\
\hline $20: 5 n-3$ & 0.07 & 0.01 & $1.36 \S$ & 0.17 & 0.03 & 0.01 & 0.11 & 0.02 & $1.68 \S$ & 0.13 & 0.15 & 0.07 \\
\hline $22: 6 n-3$ & 1.57 & $0 \cdot 18$ & $8.01 \S$ & 1.01 & 1.08 & 0.20 & $2 \cdot 99^{*}$ & 0.38 & $12 \cdot 84 \S^{*}$ & $1 \cdot 30$ & 1.87 & 0.52 \\
\hline $20: 4 n-6: 22: 6 n-3$ ratio & 0.97 & 0.06 & $0.10 \S$ & 0.01 & $2 \cdot 38 \S$ & 0.25 & $0.63^{*}$ & 0.06 & $0.06 \S^{*}$ & 0.01 & $1.28 \S^{*}$ & 0.10 \\
\hline$\Sigma$ SFA & 38.82 & 0.78 & $29 \cdot 72 \dagger$ & 0.61 & $26 \cdot 66 \dagger$ & 2.06 & $39 \cdot 33$ & 1.09 & $27 \cdot 23 \dagger$ & 1.93 & $23.72 \dagger$ & 0.58 \\
\hline$\Sigma$ MUFA $\|$ & $42 \cdot 33$ & 0.83 & 39.52 & 1.18 & $29.22 \S$ & 0.86 & 39.47 & $1 \cdot 10$ & $36 \cdot 86$ & 0.95 & $29.30 \S$ & 0.41 \\
\hline$\sum n-6$ PUFA\| & $16 \cdot 01$ & 0.32 & 18.05 & 0.63 & $41 \cdot 86 \S$ & 1.21 & $16 \cdot 24$ & 0.59 & $17 \cdot 06$ & 0.39 & $43.62 \S$ & 0.71 \\
\hline$\sum n-3$ PUFA $\|$ & $2 \cdot 83$ & 0.20 & $12 \cdot 69 \S$ & 1.59 & $2 \cdot 24$ & 0.28 & 4.94 & 0.58 & $18 \cdot 82 \S^{*}$ & $1 \cdot 84$ & 3.34 & 0.69 \\
\hline Unsaturation index & 93.76 & 1.99 & $147 \cdot 76 \S$ & 7.00 & $130 \cdot 91 \S$ & $4 \cdot 17$ & $104.58^{*}$ & 3.87 & $179 \cdot 11 \S^{*}$ & $9 \cdot 83$ & $140.37 \S$ & 4.01 \\
\hline \multicolumn{13}{|l|}{ Phospholipids } \\
\hline $16: 0$ & $10 \cdot 07$ & 0.34 & 10.92 & 0.29 & $9.08 \S$ & 0.24 & $11.55^{*}$ & 0.37 & $12 \cdot 43$ & 0.60 & $10 \cdot 91^{*}$ & 0.42 \\
\hline $18: 0$ & $26 \cdot 04$ & 0.78 & $25 \cdot 50$ & 1.76 & $26 \cdot 30^{\circ}$ & 1.65 & $25 \cdot 60$ & 0.70 & $25 \cdot 29$ & 1.01 & $26 \cdot 25$ & 1.30 \\
\hline $18: 1 n-9$ & 3.76 & $0 \cdot 11$ & $3.34 \S$ & 0.08 & 3.69 & 0.09 & $3 \cdot 70$ & $0 \cdot 19$ & $3 \cdot 31$ & 0.06 & $3 \cdot 27^{*}$ & 0.12 \\
\hline $18: 2 n-6$ & $20 \cdot 13$ & 1.36 & 20.54 & 0.70 & $25 \cdot 81 \S$ & 1.56 & $17 \cdot 75$ & 0.15 & $16 \cdot 63^{*}$ & 0.36 & $24.43 \S$ & 0.75 \\
\hline $20: 4 n-6$ & $22 \cdot 08$ & 0.20 & $11 \cdot 61 \S$ & 0.50 & 21.44 & 0.87 & $19 \cdot 91^{*}$ & 0.47 & $11 \cdot 18 \S$ & 0.54 & $18 \cdot 96^{*}$ & 0.51 \\
\hline $20: 5 n-3$ & 0.17 & 0.03 & $2.89 \S$ & 0.18 & 0.04 & 0.01 & 0.20 & 0.04 & $2.74 \S$ & 0.15 & 0.06 & 0.01 \\
\hline $22: 6 n-3$ & $10 \cdot 41$ & 0.39 & $17 \cdot 31 \S$ & 0.86 & $7 \cdot 12 \S$ & 0.54 & $12 \cdot 52^{*}$ & 0.76 & $19 \cdot 83 \S$ & 0.88 & $8.91 \S^{\star}$ & 0.43 \\
\hline $20: 4 n-6: 22: 6 n-3$ ratio & $2 \cdot 14$ & 0.08 & $0.67 \S$ & 0.01 & $3.06 \S$ & 0.13 & $1.62^{*}$ & 0.07 & $0.56 \S^{*}$ & 0.01 & $2 \cdot 15 \S^{*}$ & 0.09 \\
\hline$\Sigma$ SFA $\|$ & $36 \cdot 21$ & 1.03 & $36 \cdot 60$ & 2.03 & 35.52 & 1.86 & $37 \cdot 25$ & 1.03 & 36.73 & 1.49 & 36.88 & 2.06 \\
\hline$\Sigma$ MUFA\| & 6.99 & $0 \cdot 17$ & $7.93 \S$ & 0.18 & $6 \cdot 14 \S$ & 0.17 & $7 \cdot 33$ & 0.32 & $8 \cdot 30 \S$ & 0.19 & $5 \cdot 87 \S$ & 0.17 \\
\hline$\sum n-6$ PUFA $\|$ & 44.12 & 1.46 & $33.13 \S$ & 0.97 & $49 \cdot 77 \S$ & 1.80 & $40 \cdot 38$ & 0.50 & $29 \cdot 31 \S$ & 0.60 & $46.96 \S$ & 1.67 \\
\hline$\sum n-3$ PUFA $\|$ & $12 \cdot 66$ & 0.55 & $22.32 \S$ & 1.05 & $8.54 \S$ & 0.75 & $15 \cdot 01$ & 0.89 & $25 \cdot 64 \S^{\star}$ & 1.04 & $10 \cdot 26 \S$ & 0.59 \\
\hline Unsaturation index & 216.49 & $2 \cdot 83$ & $227 \cdot 46$ & $8 \cdot 85$ & $203 \cdot 27$ & 6.94 & $220 \cdot 92$ & $6 \cdot 61$ & 233.91 & $8 \cdot 38$ & $204 \cdot 20 \ddagger$ & $5 \cdot 70$ \\
\hline \multicolumn{13}{|l|}{ Diacylglycerols } \\
\hline $16: 0$ & 27.97 & 1.80 & 24.77 & $1 \cdot 19$ & $23 \cdot 86$ & 1.90 & 23.53 & 1.45 & 21.44 & 1.37 & $21 \cdot 11$ & 1.53 \\
\hline $18: 0$ & 23.76 & 2.46 & 19.51 & 1.78 & 21.37 & $2 \cdot 12$ & 24.83 & $2 \cdot 18$ & 18.97 & $2 \cdot 23$ & $22 \cdot 44$ & 2.32 \\
\hline $18: 1 n-9$ & $20 \cdot 85$ & $4 \cdot 11$ & 18.85 & $2 \cdot 51$ & $18 \cdot 63$ & 2.44 & 19.97 & 3.07 & $18 \cdot 57$ & 3.90 & 14.80 & $2 \cdot 66$ \\
\hline $18: 2 n-6$ & $6 \cdot 42$ & 0.44 & 8.59 & 1.00 & $14 \cdot 37 \S$ & $2 \cdot 25$ & 8.46 & 0.67 & $10 \cdot 22$ & 0.97 & $16 \cdot 15 \S$ & 1.62 \\
\hline $20: 4 n-6$ & 4.20 & 1.04 & 3.63 & 0.57 & 4.94 & 0.94 & 5.85 & 1.09 & 5.85 & 1.45 & $8.56^{*}$ & 1.69 \\
\hline $20: 5 n-3$ & $1 \cdot 18$ & 0.91 & $2 \cdot 15$ & $1 \cdot 22$ & 1.76 & 1.42 & 0.38 & 0.07 & $1.59 \S$ & 0.33 & 0.54 & 0.23 \\
\hline $22: 6 n-3$ & $2 \cdot 11$ & 0.43 & $3.69 \S$ & 0.61 & 2.04 & 0.22 & 3.29 & 0.44 & $6 \cdot 01 \S^{\star}$ & 0.98 & $3 \cdot 70$ & 0.57 \\
\hline $20: 4 n-6: 22: 6 n-3$ ratio & 1.99 & 0.27 & 0.95 & 0.15 & $2.46 \S$ & 0.43 & 1.65 & 0.20 & $1 \cdot 12$ & 0.22 & $2 \cdot 39 \ddagger$ & 0.35 \\
\hline$\Sigma$ SFA\| & $53 \cdot 70$ & $2 \cdot 84$ & $46 \cdot 26$ & 1.54 & $46 \cdot 67$ & 2.50 & $49 \cdot 69$ & $2 \cdot 35$ & 41.51 & 1.27 & 45.07 & 1.43 \\
\hline$\Sigma$ MUFA\| & $30 \cdot 36$ & 3.59 & $32 \cdot 91$ & $2 \cdot 26$ & $27 \cdot 58$ & 2.07 & $29 \cdot 54$ & $2 \cdot 67$ & 31.69 & 3.25 & $22 \cdot 75$ & $2 \cdot 64$ \\
\hline$\sum n-6$ PUFA $\|$ & 11.63 & 1.50 & $13 \cdot 61$ & 1.57 & $21 \cdot 04 \S$ & $2 \cdot 82$ & $15 \cdot 81$ & 1.50 & $17 \cdot 25$ & $2 \cdot 05$ & $26.53 \S$ & $2 \cdot 65$ \\
\hline$\sum n-3$ PUFA\| & 4.28 & 0.86 & $7 \cdot 20$ & 1.28 & 4.68 & 1.35 & 4.94 & 0.51 & $9.53 \S$ & $1 \cdot 38$ & $5 \cdot 63$ & 0.65 \\
\hline Unsaturation index & $75 \cdot 16$ & 5.74 & 88.90 & $5 \cdot 20$ & $95.48 \dagger$ & 5.39 & $85 \cdot 14$ & 5.45 & $102.25 \dagger$ & $6 \cdot 12$ & $108.07 \dagger$ & 6.57 \\
\hline
\end{tabular}

${ }^{*}$ Mean value was significantly different from that of the corresponding normoxic group $(P<0.05)$.

† Mean value was significantly different from that of the corresponding lard dietary group $(P<0.05)$.

$\ddagger$ Mean value was significantly different from that of the corresponding fish oil dietary group $(P<0.05)$.

$\S$ Mean value was significantly different from those of the other dietary groups $(P<0.05)$.

$\| \Sigma$ SFA, $\Sigma$ MUFA, $\Sigma n$-6 PUFA and $\Sigma n$-3 PUFA comprise of all fatty acids analysed.

I The unsaturation index is calculated as mol \% of individual unsaturated fatty acids multiplied by the number of double bonds 
the unsaturation index decreased in both groups. In the maize oil dietary group, the increase in the proportion of $18: 2 n-6$ was observed after adaptation to $\mathrm{CIH}$.
In serum CE, the proportions of individual SFA and MUFA were very low as compared with the other lipids analysed. The distinguishing feature of CE was the high level of $20: 4 n-6$ in
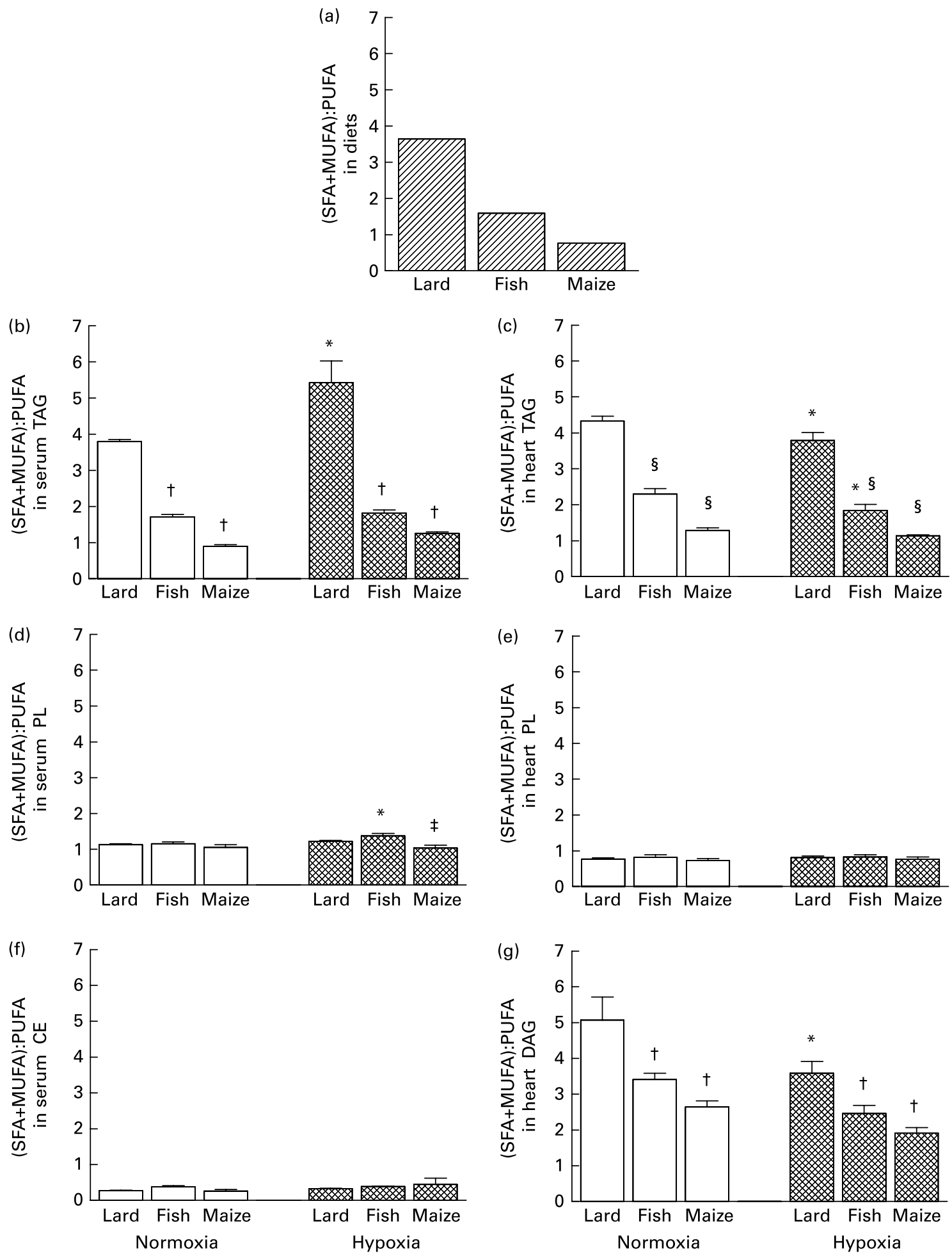

Fig. 1. The (SFA + MUFA):PUFA ratio in the diet (a), serum TAG (b), heart TAG (c), serum phospholipids (PL) (d), heart PL (e), serum cholesteryl esters (CE) $(\mathrm{f})$ and heart diacylglycerols (DAG) (g) of normoxic rats and chronically hypoxic rats fed a lard, fish oil or maize oil diet. Values are means of five animals per group, with standard errors represented by vertical bars. ${ }^{*}$ Mean value was significantly different from that of the corresponding normoxic group $(P<0.05)$. $†$ Mean value was significantly different from that of the corresponding lard dietary group $(P<0.05)$. $¥$ Mean value was significantly different from that of the corresponding fish oil dietary group $(P<0.05)$. § Mean value was significantly different from those of the other dietary groups $(P<0.05)$. 

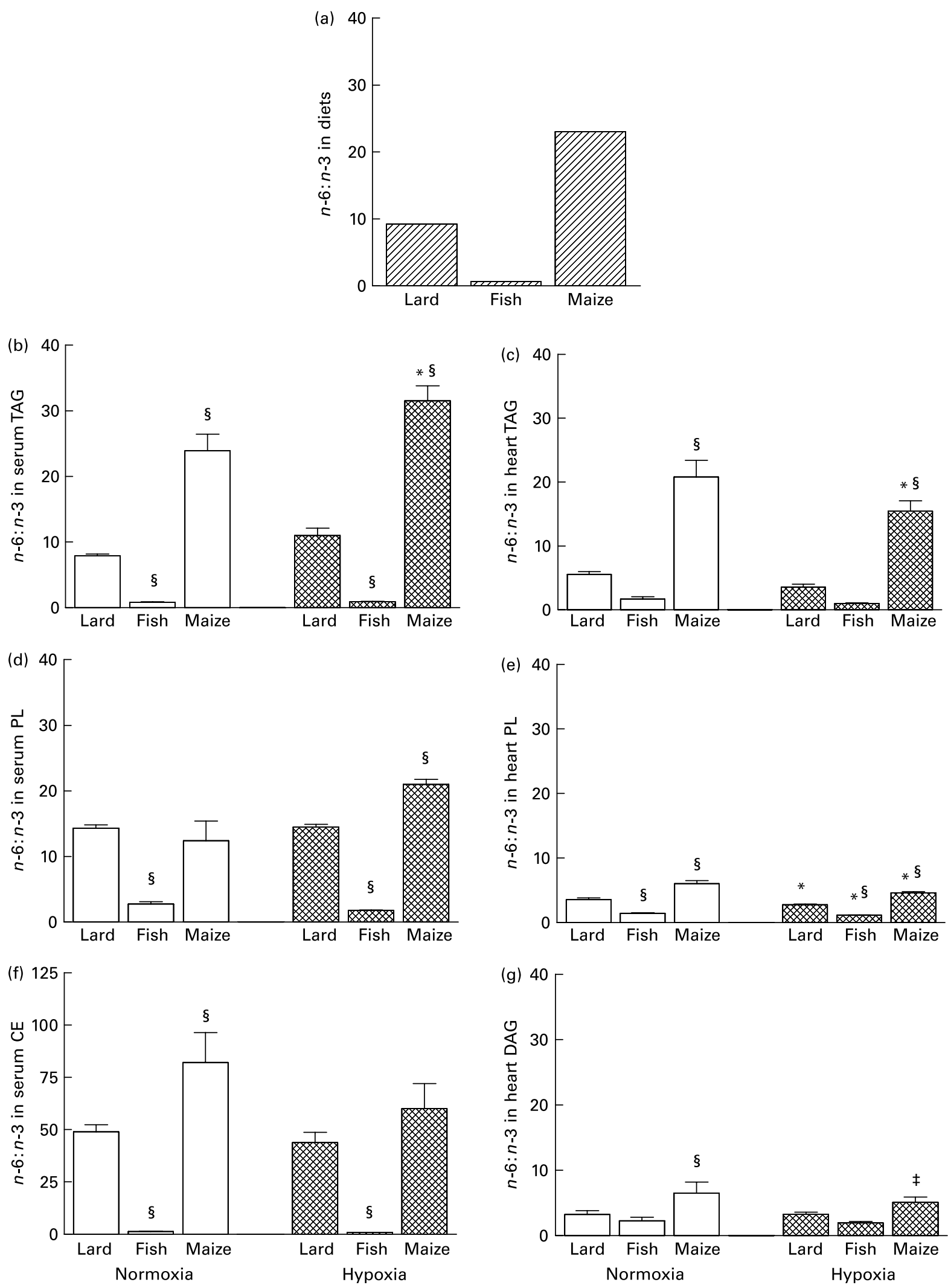

Fig. 2. The $n-6: n-3$ PUFA ratio in the diet (a), serum TAG (b), heart TAG (c), serum phospholipids (PL) (d), heart PL (e), serum cholesteryl esters (CE) $(f)$ and heart diacylglycerols (DAG) (g) of normoxic rats and chronically hypoxic rats fed a lard, fish oil or maize oil diet. Note that the vertical axis of the graph of serum CE (f) has a different scale. Values are means of five animals per group, with standard errors represented by vertical bars. ${ }^{*}$ Mean value was significantly different from that of the corresponding normoxic group $(P<0.05)$. $¥$ Mean value was significantly different from that of the corresponding fish oil dietary group $(P<0.05)$. $\S$ Mean value was significantly different from those of the other dietary groups $(P<0.05)$. 
Table 4. Level of conjugated dienes and antioxidative enzyme activities in myocardial homogenates of normoxic and chronically hypoxic rats fed a lard, fish oil or maize oil diet

(Mean values with their standard errors for six hearts per group)

\begin{tabular}{|c|c|c|c|c|c|c|c|c|c|c|c|c|}
\hline & \multicolumn{6}{|c|}{ Normoxia } & \multicolumn{6}{|c|}{ Hypoxia } \\
\hline & \multicolumn{2}{|c|}{ Lard } & \multicolumn{2}{|c|}{ Fish oil } & \multicolumn{2}{|c|}{ Maize oil } & \multicolumn{2}{|c|}{ Lard } & \multicolumn{2}{|c|}{ Fish oil } & \multicolumn{2}{|c|}{ Maize oil } \\
\hline & Mean & SEM & Mean & SEM & Mean & SEM & Mean & SEM & Mean & SEM & Mean & SEM \\
\hline Conjugated dienes (nmol/g, w/w) & 310 & 25 & $442 \S$ & 19 & 344 & 20 & 297 & 19 & $435 \S$ & 37 & 358 & 17 \\
\hline Catalase activity (U/mg protein) & $19 \cdot 6$ & $2 \cdot 1$ & $18 \cdot 2$ & 1.3 & 14.9 & 1.1 & 20.0 & 1.2 & $25 \cdot 6^{\star}$ & $2 \cdot 1$ & $23 \cdot 3^{\star}$ & 2.5 \\
\hline GPX activity (U/mg protein) & 1.31 & 0.20 & $1 \cdot 13$ & 0.18 & 0.87 & 0.09 & 1.30 & 0.11 & 1.06 & 0.17 & 1.26 & 0.15 \\
\hline SOD activity (U/mg protein) & 243 & 43 & 248 & 37 & 205 & 29 & 209 & 13 & 214 & 15 & 182 & 15 \\
\hline
\end{tabular}

GPX, glutathione peroxidase; SOD, superoxide dismutase.

${ }^{*}$ Mean value was significantly different from that of the corresponding normoxic group $(P<0.05)$.

$\S$ Mean value was significantly different from those of the other dietary groups $(P<0.05)$.

the lard and maize oil dietary groups, representing about $50 \%$ of total fatty acids. The fish oil diet replaced $18: 2 n-6$ and $20: 4 n-6$ by the equimolar proportion of $20: 5 n-3$ and $22: 6 n-3$. CIH further increased $20: 5 n-3$ at the expense of $18: 2 n-6$ and $20: 4 n-6$ in the fish oil dietary group.

Table 3 presents the fatty acid composition of heart lipids. Both PUFA dietary groups had a lower proportion of SFA $(16: 0,18: 0)$ and a higher unsaturation index in heart TAG as compared with the lard group. The MUFA proportion was the lowest and that of $n-6$ PUFA the highest one in the maize oil group, compared with other dietary groups. The fish oil group exhibited a higher proportion of $n$-3 PUFA compared with other dietary groups. CIH increased 22:6n-3 and the unsaturation index in the lard and fish oil groups. The $20: 4 n-6: 22: 6 n-3$ ratio was decreased by $\mathrm{CIH}$ in all dietary groups.

In heart PL of the fish oil group, the accumulation of $20: 5 n-3$ and $22: 6 n-3$ at the expense of $20: 4 n-6$ was observed as compared with the other two dietary groups. Adaptation to CIH increased 16:0, 22:6n-3 and decreased $20: 4 n-6$ proportions in the lard and maize oil dietary groups, while it decreased $18: 2 n-6$ and increased total $n$-3 PUFA proportions in the fish oil group. The $20: 4 n-6: 22: 6 n-3$ ratio was decreased by $\mathrm{CIH}$ in all dietary groups.

In heart DAG, SFA and MUFA were the most prominent fatty acids. The proportion of $22: 6 n-3$ was higher in the fish oil group whereas $18: 2 n-6$ was particularly enhanced in the maize oil group as compared with the other two dietary groups. CIH further increased the $22: 6 n-3$ or $20: 4 n-6$ proportions in the fish oil or maize oil dietary groups, respectively.

In serum TAG and heart TAG and DAG, the (SFA + MUFA):PUFA ratio (Fig. 1) closely followed that of the corresponding fed diet. On the other hand, this ratio remained unaffected by diets in serum PL and CE, as well as in heart PL. The (SFA + MUFA):PUFA ratio was considerably lower in serum PL, serum CE and heart PL as compared with other lipid classes due to the relatively high proportion of total PUFA. CIH increased the (SFA + MUFA):PUFA ratio in serum TAG of the lard group and in serum PL of the fish oil group. In contrast, $\mathrm{CIH}$ induced a decrease of this ratio in heart TAG and DAG but had no effect in serum $\mathrm{CE}$ and heart PL.

All lipid classes tended to reflect the $n-6: n-3$ ratio of the corresponding fed diet (Fig. 2). The ratio was the highest in the maize oil dietary group and the lowest in the fish oil group. Nevertheless, the difference in these ratios among the groups was less pronounced in the heart PL and DAG as compared with other lipids. CIH induced a decrease of the $n-6: n-3$ ratio in all lipid classes, except for serum TAG.

\section{Conjugated dienes and antioxidative enzymes}

The fish oil diet increased the level of conjugated dienes in both normoxic and hypoxic myocardium as compared with the corresponding lard or maize oil dietary groups. $\mathrm{CIH}$ had no additional effect (Table 4). However, CIH enhanced catalase activity in the hearts of rats fed both PUFA diets compared with the lard group. Activities of SOD and GPX were affected by neither lipid diets nor CIH (Table 4).

\section{Discussion}

Effect of diets and chronic intermittent hypoxia on the fatty acid composition in serum and heart lipids

In agreement with other studies ${ }^{(17)}$, we confirmed that the $n-6: n-3$ PUFA ratio in all serum and heart lipids was shifted in favour of the predominant PUFA class in the diet. $\mathrm{CIH}$ induced a decrease of the $n-6: n-3$ PUFA ratio due to the $22: 6 n-3$ enrichment at the expense of $20: 4 n-6$ in all heart lipids, independent of the diet. This effect was the most consistent in heart PL. Whereas the (SFA + MUFA):PUFA ratio in serum and heart TAG and DAG reflected the ratio of the corresponding diet, in serum PL and CE and heart PL it was kept relatively constant regardless of dietary interventions or $\mathrm{CIH}$.

The primary role of TAG is the transport of fatty acids into tissues providing a store of energy. In the present study, fatty acid profiles of serum and heart TAG closely followed those of the diets in line with the fact that samples were collected from fed rats, i.e. chylomicrons were the predominant form of circulating TAG-rich lipoproteins. It is generally accepted that fatty acids derived from the hydrolysis of TAG-rich chylomicrons may be the primary source of fatty acids utilised by the heart, particularly in the fed state ${ }^{(18)}$.

CE represent the transport form of cholesterol in the blood that is supplied to the heart, which has low cholesterol biosynthetic capacity ${ }^{(19)}$. The present results show that nearly a half of the overall fatty acid content in $\mathrm{CE}$ of the lard and maize oil 
groups comprises $20: 4 n-6$ while in the fish oil group this PUFA is replaced to a large extent by $20: 5 n-3$. It has been reported that $20: 5 n-3$ is the preferred substrate both for lysophosphatidylcholine acyltransferase, leading to the formation of phosphatidylcholine in the rat liver ${ }^{(20)}$, and for plasma lecithin:cholesterol acyltransferase which transfers PUFA from plasma phosphatidylcholine to $\mathrm{CE}^{(21)}$. This may explain the partial replacement of $20: 4 n-6$ with $20: 5 n-3$ in plasma $\mathrm{CE}$ of the fish oil dietary group in agreement with results of Garg et al. ${ }^{(22)}$. CIH further increased the incorporation of $20: 5 n-3$ into CE of the fish oil group, probably due to the enhanced supply of $20: 5 n-3$ from serum PL (i.e. phosphatidylcholine).

Serum and heart PL contained substantially higher proportions of $18: 0$ and $20: 4 n-6$ than the diets. It has been reported that a large proportion of $18: 0$ and $20: 4 n-6$ is incorporated into PL due to the preferential affinity of acyltransferases and transacylases for these fatty acids during the fatty acid remodelling process associated with PL de novo synthesis in the rat liver ${ }^{(23)}$. In the fish oil group, 20:5n-3 was preferentially incorporated into serum PL and CE whereas the accumulation of $22: 6 n-3$ was favoured in all heart lipids despite nearly equimolar proportions of these two $n-3$ PUFA in the diet. The preferential incorporation of $22: 6 n-3$ into myocardial lipids has been reported in other studies on rodents $^{(24)}$ and human subjects ${ }^{(25)}$. CIH increased the accumulation of $22: 6 n-3$ at the expense of $20: 4 n-6$ in myocardial PL of the lard and maize oil groups. However, in the fish oil group, CIH stimulated the accumulation of $22: 6 n-3$ in heart PL at the expense of $18: 2 n-6$ without any compensatory effect on $20: 4 n-6$. It is evident that $\mathrm{CIH}$-adapted myocardium tends to preserve the relatively low level of $20: 4 n-6$ in PL that was already reduced by the fish oil diet.

The shifts in the $n-6$ and $n-3$ PUFA proportions in heart PL caused by diets and $\mathrm{CIH}$ were qualitatively similar to those observed in rats fed different PUFA-enriched diets and chronically treated with high doses of catecholamines ${ }^{(26)}$. In line with this observation, exposure of rats to $\mathrm{CIH}$ was associated with transiently increased adrenergic activity and elevated plasma levels of catecholamines ${ }^{(27,28)}$. Thus, the fatty acid remodelling in PL induced by $\mathrm{CIH}$ could be explained by stress-dependent hormonal modulation of enzyme activities involved in PL acyl remodelling. PL deacylation was accelerated due to the activation of phospholipase A2 in cardiomyocytes exposed to hypoxia ${ }^{(29,30)}$. It is likely that this enzyme which preferentially hydrolyses $20: 4 n-6$ from the sn2 position of membrane $\mathrm{PL}^{(31)}$ and acyl-CoA synthase with preferential affinity for $22: 6 n-3^{(32)}$ could contribute to the $22: 6 n-3$ accumulation in heart PL by the deacylation-reacylation cycle observed after $\mathrm{CIH}$.

Our observation that DAG comprised a high proportion of SFA and MUFA but a low proportion of PUFA as compared with other lipids regardless of dietary interventions is in agreement with several reports ${ }^{(33-36)}$. The fish oil diet increased the $22: 6 n-3$ proportion in DAG compared with other diets. This observation is in line with the results obtained in fish oil-supplemented mice and $\operatorname{dogs}{ }^{(37,38)}$. DAG are important endogenous lipid activators of $\mathrm{PKC}^{(39)}$. Besides a direct interaction of this lipid with PKC, changes induced in the membrane lipid structure by DAG and other lipids favour the binding and activation of $\mathrm{PKC}^{(40)}$. It has been shown that DAG enriched with
$20: 5 n-3$ or $22: 6 n-3$ were less efficient activators of PKC than DAG containing $20: 4 n-6^{(41)}$. Judé et al. ${ }^{(38)}$ observed a lower activation of PKC $\delta$ and PKC $\varepsilon$ in the hearts of fish oil-supplemented dogs than in those fed the standard diet. In line with the results mentioned above, Hlavackova et al. demonstrated a lower abundance of PKC $\delta$ in the particulate fraction from normoxic hearts of rats fed the fish oil diet, together with the larger myocardial infarct size compared with the maize oil dietary group ${ }^{(10)}$. On the other hand, adaptation to $\mathrm{CIH}$ that further increased the $22: 6 n-3$ proportion in heart DAG of the fish oil group stimulated the expression of PKC $\delta$ and had a protective effect on infarct size. These results suggest that the impact of changes in the PUFA composition of DAG on PKC activation or inactivation in connection with cardiac ischaemic tolerance is complex and may differ in normoxic and chronically hypoxic hearts. CIH also increased $20: 4 n-6$ in DAG of the maize oil group and tended to increase it in the lard and fish oil groups in contrast with heart TAG and PL where 20:4n-6 either decreased or remained unchanged. The fact that $\mathrm{CIH}$ specifically increased the accumulation of $20: 4 n-6$ in myocardial phosphatidylcholine ${ }^{(9)}$ offers the explanation that phosphatidylcholine could be an important source of DAG under the $\mathrm{CIH}$ conditions. In accordance with this assumption, it has been reported that DAG generated from phosphatidylcholine by phospholipase $\mathrm{D}$ is involved in ischaemic preconditioning ${ }^{(42,43)}$. Murase et al. ${ }^{(35)}$ found an increased DAG content as well as an increased proportion of $20: 4 n-6$ in DAG of preconditioned rat hearts, supporting the involvement of this signalling lipid in the cardioprotection.

There is a general consensus that $n-3$ PUFA, particularly $20: 5 n-3$ and $22: 6 n-3$, exert powerful anti-arrhythmic effects $^{(44)}$ resulting from their multiple actions. It was shown, for example, that both $20: 5 n-3$ and $22: 6 n-3$ decreased cardiac susceptibility to adrenergic stimulation ${ }^{(45)}$, prevented disturbances of membrane transport and ionic homeostasis $^{(46,47)}$, decreased phosphoinositides production ${ }^{(48)}$, altered the fatty acid composition of DAG and led to differential PKC activation ${ }^{(38)}$. Therefore, the enrichment of $20: 5 n-3$ and $22: 6 n-3$ in serum and heart lipids demonstrated in the present study could contribute to the anti-arrhythmic effect of $\mathrm{CIH}$ observed under ischaemia-reperfusion conditions that was most pronounced in the fish oil group ${ }^{(10)}$.

\section{Effect of diets and chronic intermittent hypoxia on myocardial redox status}

The increased level of conjugated dienes in the myocardium of fish oil-supplemented rats is in agreement with the increased susceptibility of $n-3$ PUFA to oxidative stress, compared with the $n-6$ PUFA class, probably due to the greater presence of double bonds ${ }^{(49)}$. Conjugated dienes are one of the primary metabolic intermediate products of lipoperoxidation $^{(50,51)}$. Our finding of increased levels of conjugated dienes in the myocardium of the fish oil dietary group corresponds to the largest myocardial infarct size observed in these animals. On the other hand, the same fish oil group exhibited the lowest incidence and severity of ventricular $\operatorname{arrhythmias}^{(10)}$. This is in line with the suggestion of Judé et al. ${ }^{(52)}$ that an oxidation product of $22: 6 n-3$ could effectively protect against arrhythmias rather than $22: 6 n-3$ itself. 
Despite the increased formation of conjugated dienes in the fish oil group, we did not notice any change in the activities of SOD, GPX or catalase. Likewise, Nageswari et al. ${ }^{(53)}$ did not observe any effect of a fish oil diet on antioxidative enzyme activities in rat hearts despite the increased level of hydroperoxides. In contrast, Diniz et al. ${ }^{(54)}$ have shown that both $n-3$ and n-6 PUFA diets decreased the activities of SOD and catalase and increased the activity of GPX in rat hearts. Under similar experimental conditions, a decrease in activities of myocardial SOD and GPX was detected in another study ${ }^{(55)}$. This inconsistency of available data regarding the effects of lipid dietary supplementation may be due to the variability of experimental models, fatty acid composition of diets and their intake level.

Recently we demonstrated that $\mathrm{CIH}$ was associated with increased oxidative stress as evidenced by decreased myocardial reduced:oxidised glutathione ratio and elevated concentration of lipofuscin-like pigments ${ }^{(8)}$. The present study did not detect any significant effect of $\mathrm{CIH}$ on the myocardial level of conjugated dienes in any dietary group. This can be explained by the fact that these compounds are one of the first temporarily detectable unstable markers of lipoperoxidation. It seems likely that changes in the level of conjugated dienes could have appeared at earlier stages of hypoxic adaptation. $\mathrm{CIH}$ increased the activity of catalase in the hearts of the fish oil and maize oil groups as compared with the lard-fed group. This suggests that oxidative stress associated with CIH was probably higher in both PUFA dietary groups than in the lard group. In line with our observation, $\mathrm{CIH}$ enhanced the activity of catalase in the hearts of rats kept on a standard diet ${ }^{(56)}$. On the other hand, myocardial activities of catalase and GPX did not differ between normoxic and chronically hypoxic young rats whereas the SOD activity was decreased in the hypoxic group ${ }^{(57)}$. Although total SOD and GPX remained unchanged in the myocardial homogenate of $\mathrm{CIH}$ animals, we cannot exclude that their activities increased in specific subcellular compartments, such as mitochondria, that are involved in reactive oxygen species generation.

We conclude that both dietary interventions as well as $\mathrm{CIH}$ had systemic effects leading to distinct changes in the fatty acid profile in serum and heart lipids. In particular, membrane PL maintained SFA, MUFA and total PUFA proportions constant independently of diet and CIH. This is the evidence of a remarkable regulatory ability of membranes to maintain a stable milieu that is necessary for a proper function of membrane proteins. On the other hand, the $n-6: n-3$ PUFA ratio was influenced to various extents by either the dietary PUFA supply or CIH in all lipid classes. These changes may affect myocardial adaptive responses under various physiological and pathophysiological conditions, such as those associated with ischaemia-reperfusion and oxidative stress.

\section{Acknowledgements}

The present study was supported by the Ministry of Education, Youth and Sports of the Czech Republic (grant no. 0021620858) and the Czech Science Foundation (grant no. 305/07/0875).
P. B. measured enzyme activities and participated in drafting the manuscript; J. J. was responsible for lipid analysis; M. H. measured the level of conjugated dienes; J. N. and F. K. performed animal experiments and tissue sampling and participated in preparation of the manuscript; B. S. was responsible for fatty acid analysis; O. N. and F. N. were responsible for interpretation of the results and preparation of the manuscript.

There are no conflicts of interest.

\section{References}

1. Escriba PV (2006) Membrane-lipid therapy: a new approach in molecular medicine. Trends Mol Med 12, 34-43.

2. Nair SD, Leitch JW, Falconer J, et al. (1997) Prevention of cardiac arrhythmia by dietary $(n-3)$ polyunsaturated fatty acids and their mechanism of action. $J$ Nutr 127, 383-393.

3. Demaison L \& Moreau D (2002) Dietary $n-3$ polyunsaturated fatty acids and coronary heart disease-related mortality: a possible mechanism of action. Cell Mol Life Sci 59, 463-477.

4. Teres S, Barcelo-Coblijn G, Benet M, et al. (2008) Oleic acid content is responsible for the reduction in blood pressure induced by olive oil. Proc Natl Acad Sci U S A 105, $13811-13816$

5. Isensee H \& Jacob R (1994) Differential effects of various oil diets on the risk of cardiac arrhythmias in rats. $J$ Cardiovasc Risk 1, 353-359.

6. Das UN (2006) Essential fatty acids: biochemistry, physiology and pathology. Biotechnol J 1, 420-439.

7. Kolar F \& Ostadal B (2004) Molecular mechanisms of cardiac protection by adaptation to chronic hypoxia. Physiol Res 53, S3-S13.

8. Kolar F, Jezkova J, Balkova P, et al. (2007) Role of oxidative stress in PKC- $\delta$ upregulation and cardioprotection induced by chronic intermittent hypoxia. Am J Physiol Heart Circ Physiol 292, $\mathrm{H} 224-\mathrm{H} 230$

9. Jezkova J, Novakova O, Kolar F, et al. (2002) Chronic hypoxia alters fatty acid composition of phospholipids in right and left ventricular myocardium. Mol Cell Biochem 232, 49-56.

10. Hlavackova M, Neckar J, Jezkova J, et al. (2007) Dietary polyunsaturated fatty acids alter myocardial protein kinase $\mathrm{C}$ expression and affect cardioprotection induced by chronic hypoxia. Exp Biol Med 232, 823-832.

11. Tvrzicka E, Vecka M, Stankova B, et al. (2002) Analysis of fatty acids in plasma lipoproteins by gas chromatographyflame ionisation detection. Quantitative aspects. Anal Chim Acta 465, 337-350.

12. Folch J, Lees M \& Sloane Stanley GH (1957) A simple method for the isolation and purification of total lipides from animal tissue. J Biol Chem 226, 497-509.

13. Aebi H (1984) Catalase in vitro. Methods Enzymol 105, $121-126$.

14. Paglia DE \& Valentine WN (1967) Studies on the quantitative and qualitative characterization of erythrocyte glutathione peroxidase. J Lab Clin Med 70, 158-169.

15. Elstner EF, Youngman RJ \& Obwald W (1983) Superoxide dismutase. In Methods of Enzymatic Analysis, 2nd ed., pp. 293-302 [HU Bergmeyer, editor]. Weinheim, Germany: Verlag Chemie.

16. Ahotupa M, Ruutu M \& Mäntylä E (1996) Simple methods of quantifying oxidation products and antioxidant potential of low density lipoproteins. Clin Biochem 29, 139-144.

17. Stubbs CD \& Smith AD (1984) The modification of mammalian membrane polyunsaturated fatty acid composition in relation 
to membrane fluidity and function. Biochim Biophys Acta 779, 89-137.

18. Augustus AS, Kako Y, Yagyu H, et al. (2003) Routes of FA delivery to cardiac muscle: modulation of lipoprotein lipolysis alters uptake of TG-derived FA. Am J Physiol Endocrinol Metab 284, E331-E339.

19. Spady DK \& Dietschy JM (1983) Sterol synthesis in vivo in 18 tissues of the monkey, guinea pig, rabbit, hamster, and rat. J Lipid Res 24, 303-315.

20. Iritani N \& Fujikawa S (1982) Competitive incorporation of dietary omega-3 and omega- 6 polyunsaturated fatty acids into the tissue phospholipids in rats. J Nutr Sci Vitaminol 28 , 621-629.

21. Holub BJ, Bakker DJ \& Skaeff CM (1987) Alterations in molecular species of cholesterol esters formed via plasma lecithin-cholesterol acyltransferase in human subjects consuming fish oil. Atherosclerosis 66, 11-18.

22. Garg ML, Wierzbicki AA, Thomson AB, et al. (1989) Omega-3 fatty acids increase the arachidonic acid content of liver cholesterol ester and plasma triacylglycerol fractions in the rat. Biochem J 261, 11-15.

23. Yamashita A, Sugiura T \& Waku K (1997) Acyltransferases and transacylases involved in fatty acid remodeling of phospholipids and metabolism of bioactive lipids in mammalian cells. J Biochem 122, 1-16.

24. Rousseau D, Héliès-Toussaint C, Moreau D, et al. (2003) Dietary $n-3$ PUFAs affect the blood pressure rise and cardiac impairments in a hyperinsulinemia rat model in vivo. Am $J$ Physiol Heart Circ Physiol 285, H1294-H1302.

25. Metcalf RG, James MJ, Gibson RA, et al. (2007) Effects of fish-oil supplementation on myocardial fatty acids in humans. Am J Clin Nutr 85, 1222-1228.

26. Benediktsdottir VE \& Gudbjarnason S (1988) Reversible alterations in fatty acid composition of heart muscle membrane phospholipids induced by epinephrine in rats fed different fats. J Lipid Res 29, 765-772.

27. Maher JT, Manchanda SC, Cymerman A, et al. (1975) Cardiovascular responsiveness to $\beta$-adrenergic stimulation and blockade in chronic hypoxia. Am J Physiol 228, 477-481.

28. Ostadal B, Kvetnansky R, Prochazka J, et al. (1984) Effect of intermittent high altitude stress on epinephrine and norepinephrine levels in the right and left ventricular myocardium of rats. In Role of Catecholamines and Other Neurotransmitters Under Stress, pp. 669-674 [E Usdin, R Kvetnansky and IJ Kopin, editors]. New York: Gordon and Breach.

29. Kawaguchi H, Shoki M, Iizuka K, et al. (1991) Phospholipid metabolism and prostacyclin synthesis in hypoxic myocytes. Biochim Biophys Acta 1094, 161-167.

30. Grynberg A, Nalbone G, Degois M, et al. (1988) Activities of some enzymes of phospholipid metabolism in cultured rat ventricular myocytes in normoxic and hypoxic conditions. Biochim Biophys Acta 958, 24-30.

31. Nalbone G, Grynberg A, Chevalier A, et al. (1990) Phospholipase A activity of cultured rat ventricular myocyte is affected by the nature of cellular polyunsaturated fatty acids. Lipids $\mathbf{2 5}$, 301-306.

32. Bouroudian M, Nalbone G, Grynberg A, et al. (1990) In vitro study of docosahexaenoic acid incorporation into phosphatidylcholine by enzymes of rat heart. Mol Cell Biochem 93, $119-128$.

33. Hamplova B, Novakova O, Tvrzicka E, et al. (2005) Protein kinase $\mathrm{C}$ activity and isoform expression during early postnatal development of rat myocardium. Cell Biochem Biophys 43, $105-117$.

34. Nasa Y, Sakamoto Y, Sanbe A, et al. (1997) Changes in fatty acid compositions of myocardial lipids in rats with heart failure following myocardial infarction. Mol Cell Biochem 176, 179-189.

35. Murase K, Okumura K, Hayashi K, et al. (2000) Measurements of 1,2-diacylglycerol and ceramide in hearts subjected to ischemic preconditioning. Life Sci 66, 1491-1500.

36. Eskildsen-Helmond YE, Hahnel D, Reinhardt U, et al. (1998) Phospholipid source and molecular species composition of 1,2-diacylglycerol in agonist-stimulated rat cardiomyocytes. Cardiovasc Res 40, 182-190.

37. Takahashi R, Okumura K, Asai T, et al. (2005) Dietary fish oil attenuates cardiac hypertrophy in lipotoxic cardiomyopathy due to systemic carnitine deficiency. Cardiovasc Res 68, 213-223.

38. Judé S, Martel E, Vincent F, et al. (2007) Dietary long-chain $n-3$ fatty acids modify blood and cardiac phospholipids and reduce protein kinase-C- $\delta$ and protein kinase-C- $\varepsilon$ translocation. Br J Nutr 98, 1143-1151.

39. Nishizuka Y (1992) Intracellular signaling by hydrolysis of phospholipids and activation of protein kinase C. Science $\mathbf{2 5 8}$, 607-614.

40. Goñi FM \& Alonso A (1999) Structure and functional properties of diacylglycerols in membranes. Prog Lipid Res 38, 1-48.

41. Madani S, Hichami A, Legrand A, et al. (2001) Implication of acyl chain of diacylglycerols in activation of different isoforms of protein kinase C. FASEB J 15, 2595-2601.

42. Cohen MV, Liu Y, Liu GS, et al. (1996) Phospholipase D plays a role in ischemic preconditioning in rabbit heart. Circulation 94, 1713-1718.

43. Tosaki A, Maulik N, Cordis G, et al. (1997) Ischemic preconditioning triggers phospholipase $\mathrm{D}$ signaling in rat heart. Am J Physiol 273, H1860-H1866.

44. Leaf A, Kang JX, Xiao YF, et al. (2003) Clinical prevention of sudden cardiac death by $n-3$ polyunsaturated fatty acids and mechanism of prevention of arrhythmias by $n-3$ fish oils. Circulation 107, 2646-2652.

45. Ponsard B, Durot I, Delerive P, et al. (1999) Cross-influence of membrane polyunsaturated fatty acids and hypoxia-reoxygenation on $\alpha$ - and $\beta$-adrenergic function of rat cardiomyocytes. Lipids 34, 457-466.

46. Pepe S \& McLennan PL (1996) Dietary fish oil confers direct antiarrhythmic properties on the myocardium of rats. $J$ Nutr 126, 34-42.

47. Rinaldi B, Di Pierro P, Vitelli MR, et al. (2002) Effects of docosahexaenoic acid on calcium pathway in adult rat cardiomyocytes. Life Sci 71, 993-1004.

48. Anderson KE, Du XJ, Sinclair AJ, et al. (1996) Dietary fish oil prevents reperfusion $\operatorname{Ins}(1,4,5) \mathrm{P} 3$ release in rat heart: possible antiarrhythmic mechanism. Am J Physiol 271, H1483-H1490.

49. Scislowski V, Bauchart D, Gruffat D, et al. (2005) Effects of dietary $n-6$ or $n-3$ polyunsaturated fatty acids protected or not against ruminal hydrogenation on plasma lipids and their susceptibility to peroxidation in fattening steers. J Anim Sci 83, 2162-2174.

50. Corongiu FP \& Banni S (1994) Detection of conjugated dienes by second derivative ultraviolet spectrophotometry. Methods Enzymol 233, 303-310.

51. Hendra TJ, Wickens DG, Dormandy TL, et al. (1991) Platelet function and conjugated diene concentrations in diabetic and non-diabetic survivors of acute myocardial infarction. Cardiovasc Res 25, 676-683.

52. Judé S, Bedut S, Roger S, et al. (2003) Peroxidation of docosahexaenoic acid is responsible for its effects on I TO and I SS in rat ventricular myocytes. Br J Pharmacol 139, 816-822.

53. Nageswari K, Banerjee R \& Menon VP (1999) Effect of saturated, omega- 3 and omega- 6 polyunsaturated fatty acids on myocardial infarction. $J$ Nutr Biochem 10, 338-344. 
54. Diniz YS, Cicogna AC, Padovani CR, et al. (2004) Diets rich in saturated and polyunsaturated fatty acids: metabolic shifting and cardiac health. Nutrition 20, 230-234.

55. Schimke I, Haberland A, Wirth M, et al. (1997) Influence of long-term supplementation with $\alpha$-linolenic acid on myocardial lipid peroxidation and antioxidative capacity in spontaneously hypertensive rats. Prostaglandins Leukot Essent Fatty Acids 57, 545-550.
56. Zhu WZ, Dong JW, Ding HL, et al. (2004) Postnatal development in intermittent hypoxia enhances resistance to myocardial ischemia/reperfusion in male rats. Eur J Appl Physiol 91, $716-722$.

57. Oka T, Itoi T, Terada N, et al. (2008) Change in the membranous lipid composition accelerates lipid peroxidation in young rat hearts subjected to 2 weeks of hypoxia followed by hyperoxia. Circ J 72, 1359-1366. 